\title{
Estimativa de anisotropia em poços direcionais
}

Amanda G. Pereira, Buno dos S. Silva e Ellen de N. S. Gomes, UFPA, Brazil

Copyright 2019, SBGf - Sociedade Brasileira de Geofísica

This paper was prepared for presentation at the $16^{\text {th }}$ International Congress of the Brazilian Geophysical Society, held in Rio de Janeiro, Brazil, August 19-22, 2019.

Contents of this paper were reviewed by the Technical Committee of the $16^{\text {th }}$ International Congress of The Brazilian Geophysical Society and do not necessarily represent any position of the SBGf, its officers or members. Electronic reproduction or storage of any part of this paper for commercial purposes without the written consent of The Brazilian Geophysical Society is prohibited.

\section{Abstract}

In this work, poorly anisotropic (WA) parameters (Thomsen, 1986) of an TI medium are estimated from data of slowness and polarization of $\mathrm{qP}$ waves measured in a VSP experiment with a directional well. From the WA parameter estimates, the orientation of the axis of symmetry of the medium $\mathrm{Tl}$ can be determined. This information is useful to obtain the preferential direction of flows in the reservoirs.

\section{Introdução}

Grande parte dos reservatórios de hidrocarbonetos são fraturados. Em regime quase estático, os meios fraturados comportam-se efetivamente como meios anisotrópicos (Schoenberg and Douma, 1988). A estimativa da anisotropia do meio é importante para caracterização do reservatório, pode-se por exemplo aferir a direção prefencial de fluxo.

Em Silva (2018); Zheng and Pšenčík (2002); Macambira et al. (2014); Barreto et al. (2013) os parâmetros de fraca anisotropia (parâmetros WA) são estimados a partir de aproximações de primeira ordem dos dados de vagarosidade e polarização de ondas $\mathrm{qP}$ medidos em experimentos de VSP para poços verticais.

Esse esquema de inversão independe do meio ao redor do receptor e o meio anisotrópico é considerado ser uma perturbação de primeira ordem em torno de um meio isotrópico de referência (Zheng and Pšenčík, 2002).

Este trabalho é uma extensão dessa abordagem. Os dados de vagarosidade e polarização de ondas $P$ diretas medidos em um poço direcional (com partes vertical e horizontal) são utilizados para estimar os parâmetros anisotrópicos de um meio TI (transversalmente isotrópicos). A partir dessas estimativas pretende-se estimar a orientação do eixo de simetria de meios TI.

\section{Problema direto}

Consideremos um sistema de coordenadas cartesiano $(x, y, z)$, com eixo $z$ positivo na direção que aponta de cima para baixo. Neste trabalho será considerada a formulação onde o meio anisotrópico é considerado como uma perturbação de primeira ordem de um meio isotrópico de referência. $O$ vetor de vagarosidade e polarização de uma onda $P$ que se propaga em um meio anisotropico estão relacionados linearmente com os parâmetros anisotropicos através da (Zheng and Pšenčík, 2002):

$$
\begin{aligned}
& B_{k 3}=\left(\alpha^{2}-\beta^{2}\right)\left(g_{i} e_{i}^{k}-\alpha \Delta \xi e_{1}^{k}-\alpha \Delta \zeta e_{2}^{k}-\alpha \Delta \eta e_{3}^{k}\right), k=1,2 \\
& B_{33}=-2 \alpha^{4} \xi \Delta \xi-2 \alpha^{4} \zeta \Delta \zeta-2 \alpha^{4} \eta \Delta \eta
\end{aligned}
$$

Em que: $\alpha$ e $\beta$ são, respectivamente, as velocidades das ondas $P$ e $S$ no meio isotrópico de referência. O termo $e_{i}^{k}$ faz parte do sistema vetorial formado por $e^{(1)}, e^{(2)}$ e $e^{(3)}$ no meio de referência e são escolhidos da seguinte forma (Pšenč I' k and Gajewski, 1998):

$$
\begin{aligned}
e^{1} & =D^{-1}\left(n_{1} n_{3}, n_{2} n_{3}, n_{3}^{2}-1\right) \\
e^{2} & =D^{-1}\left(-n_{2}, n_{1}, 0\right) \\
e^{2} & =D^{-1}\left(n_{1}, n_{2}, n_{3}\right)
\end{aligned}
$$

Onde $D=\sqrt{\left(n_{1}^{2}+n_{2}^{2}\right)}$. O vetor $\mathbf{n}=\left(n_{1}, n_{2}, n_{3}\right)$ em (1) e (3) é o vetor unitário perpendicular à frente de onda $P$ no meio isotrópico de referência. Os símbolos $\xi, \zeta$ e $\eta$ são as projeções do vetor de vagarosidade nas direções $x, y$ e $z$ respectivamente, $\Delta \xi, \Delta \zeta$ e $\Delta \eta$ são as perturbações destas projeções e $g_{i}$ é o vetor de polarização. Os símbolos $B_{k 3}(k=1,2)$ e $B_{33}$ são componentes da matriz de fraca anisotropia, dada por:

$$
B_{m n}=a_{i j k l} e_{i}^{m} e_{j}^{3} e_{k}^{3} e_{l}^{n}-\alpha^{2} \delta_{m n}, m, n=1,2,3 .
$$

O símbolo $a_{i j k l}$ é o tensor dos parâmetros elásticos normalizados pela densidade e $i, j, k, l=1,2,3$. A matriz de fraca anisotropia corresponde a perturbação de primeira ordem da matriz de Christoffel em torno o meio isotrópico de referência (Pšenč I' k and Gajewski, 1998).

Para que a equação (1) possa ser utilizada é necessário ter o conhecimento prévio de todas as componentes do vetor de vagarosidade e do vetor de polarização. Para determinar todas as componentes do vetor de polarização é assumido que os dados são obtidos de geofones $3 \mathrm{C}$ situado no interior dos poços. Quanto aos dados de vagarosidade, considerando o meio anisotrópico arbitrário, tem-se que só é possivel calcular a partir do tempo de trânsito do dado, a componente do vetor de vagarosidade paralela a orientação poço.

Neste trabalho, estaremos considerando um poço direcional (poço com parte vertical e parte horizontal). Para modelar essa situação estaremos utilizando a metodologia acima considerando receptores situados tanto no interior de um poço vertical quanto no interior de um poço horizontal (ver Fig. 1). Para um poço vertical, assumimos que apenas a componente vertical da 


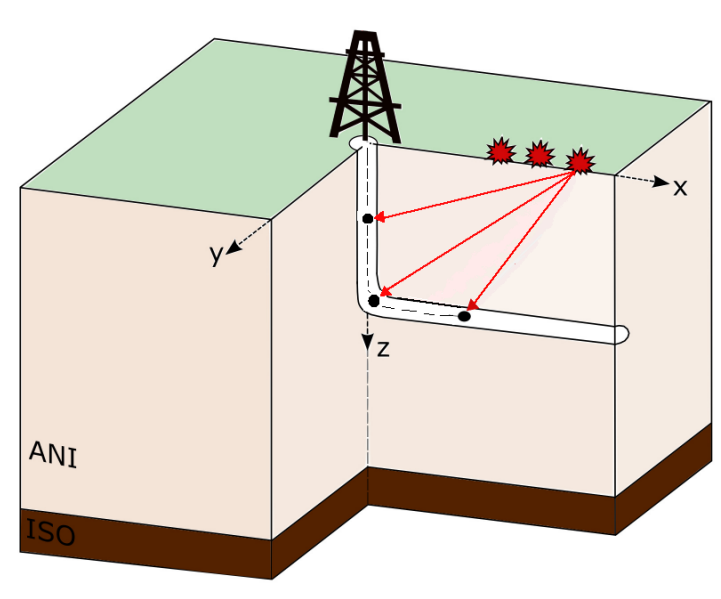

Figure 1: Configuração utilizada no experimento, constituído de dois meios, sendo o primeiro anisotrópico e o segundo isotrópico. As fontes estão na superficie e os receptores estão no interio do poço direcional. A onda $P$ direta é representada pela linha vermelha.

vagarosidade é conhecida, $\eta$, e as outras componentes da vagarosidade, $\xi$ e $\zeta$, são eliminadas da equação (1). Portanto, a relação entre polarização, a componente vertical do vetor de vagarosidade e dos parâmetros anisotrópicos do meio é dada por (Zheng and Pšenčík, 2002):

$$
D\left(\alpha^{2}-\beta^{2}\right)^{-1} B_{13}-\frac{1}{2} \alpha^{-1} \eta B_{33}=D g_{i} e_{i}^{(1)}+\alpha \Delta \eta
$$

Para um poço horizontal, assumimos que apenas componente $x$ do vetor de vagarosidade é determinada, $\xi$. Nesta caso, utilizamos a equação que relaciona a polarização e a componenete $x$ da vagarosidade aos parâmetros anisotrópicos do meio dada por (Silva, 2018):

$$
\begin{array}{r}
\left(\alpha^{2}-\beta^{2}\right)^{-1}\left(B_{13} e_{1}^{(1)}+B_{23} e_{1}^{(2)}\right)-\frac{1}{2} \alpha^{-1} B_{33} \xi= \\
g_{i}\left(e_{i}^{(1)} e_{1}^{(1)}+e_{i}^{(2)} e_{1}^{(2)}\right)-\alpha \Delta \xi .
\end{array}
$$

As equações (4) e (5) podem ser escritas na forma matricial:

$$
\mathbf{M x}=\mathbf{y},
$$

em que o vetor y é adimensional e está relacionado aos dados observados para cada par de fonte receptor (lado direito das equações (4) para o poço vertical e (5) para o poço horizontal) e possui dimensão igual ao número de observações, $N_{o b s}$. O vetor $\mathbf{x}$ está relacionado aos parâmetros $W A$ e tem dimensão igual ao número de parâmetros, $N_{\text {par }}$. A matriz $\mathbf{M}$ é de ordem $N_{o b s} x N_{\text {par }}$ e é a chamada matriz de sensibilidade. Apenas 15 parâmetros $W A$ (Weak Anisotropy) controlam a propagação da onda $P$, são eles:

$$
\begin{array}{rcl}
x_{1}=\varepsilon_{x} & x_{2}=\varepsilon_{y} & x_{3}=\varepsilon_{z} \\
x_{4}=\delta_{x} & x_{5}=\delta_{y} & x_{6}=\delta_{z} \\
x_{7}=X_{x} & x_{8}=X_{y} & x_{9}=X_{z} \\
x_{10}=\varepsilon_{15} & x_{11}=\varepsilon_{16} & x_{12}=\varepsilon_{24} \\
x_{10}=\varepsilon_{26} & x_{11}=\varepsilon_{34} & x_{12}=\varepsilon_{35}
\end{array}
$$

onde $\varepsilon, \delta$ e $X$ são os parâmetros que em conjunto com a velocidade da onda $P$ e $S$ caracterizam a propagação de ondas através de meios $W A$, denominados parâmetros de Thomsen e são grandezas adimensionais. O parâmetro $\varepsilon$ é denominado de anisotropia da onda $P$, o parâmetro $X$ é chamado de anisotropia da onda cisalhante e o parâmetro $\delta$ está relacionada pela variação da velocidade da onda $P$ em ângulos próximos a vertical (Thomsen, 1986).

Os parâmetros WA estão relacionados aos parâmetros elásticos normalizados pela densidade $\left(A_{i j}\right)$ e são calculados da seguinte maneira:

$$
\begin{array}{ccc}
\varepsilon_{x}=\frac{A_{11}-\alpha^{2}}{2 \alpha^{2}}, & \varepsilon_{y}=\frac{A_{22}-\alpha^{2}}{2 \alpha^{2}}, & \varepsilon_{z}=\frac{A_{33}-\alpha^{2}}{2 \alpha^{2}} \\
\delta_{x}=\frac{A_{13}+2 A_{55}-\alpha^{2}}{\alpha^{2}}, & \delta_{y}=\frac{A_{23}+2 A_{44}-\alpha^{2}}{\alpha^{2}}, & \delta_{z}=\frac{A_{12}+2 A_{66}-\alpha^{2}}{\alpha^{2}} \\
X_{x}=\frac{A_{14}+2 A_{56}}{\alpha^{2}}, & X_{y}=\frac{A_{25}+2 A_{46}}{\alpha^{2}}, & X_{z}=\frac{A_{36}+2 A_{45}}{\alpha^{2}}, \\
\varepsilon_{15}=\frac{A_{15}}{\alpha^{2}}, & \varepsilon_{16}=\frac{A_{16}}{\alpha^{2}}, & \varepsilon_{24}=\frac{A_{24}}{\alpha^{2}} \\
\varepsilon_{26}=\frac{A_{26}}{\alpha^{2}}, & \varepsilon_{34}=\frac{A_{34}}{\alpha^{2}}, & \varepsilon_{35}=\frac{A_{35}}{\alpha^{2}}
\end{array}
$$

\section{Problema inverso}

Os parâmetros de fraca anisotropia podem ser estimados a partir de (6), determinando-se que valores do vetor $x_{j}$ que minimizam a função:

$$
\Phi=\sum_{i=1}^{N_{\text {obs }}}\left(y_{i}-M_{i j} \widetilde{x_{j}}\right)
$$

em que: $i=1, \ldots, N_{o b s}, M_{i j}$ são os elementos da matriz de sensibilidade $M, \tilde{x_{j}}$ são as estimativas dos parâmetros $W A$ e $y_{i}$ é a i-ézima componente do vetor de observação $y$. O vetor de observação, que é constituído da componente da vagarosidade na direção do poço e o vetor de polarização são gerados sintéticamente através do ANRAY. A inversão é feita utilizando decomposição em valores singulares (Menke, 2018).

\section{Meios TI}

Meios formados por um sistema de fraturas horizontais em regime quase estático comportam-se efetivamente como um meio anisotrópico TIV (transversalmente isotrópico com eixo de simetria vetical). Da mesma forma, meios com sistema de fraturas arbitrárias comportam-se como meios TI (transversalmente isotrópicos). A direção do eixo de simetria determina a orientação das fraturas e pode ser calculado a partir dos parâmetros WA.

Para a estimativa dos parâmetros WA foi criado um modelo de $10 \mathrm{~km}$ de largura e $7 \mathrm{~km}$ de profundidade constituído de duas camadas. A primeira camada possui $5 \mathrm{~km}$ de profundidade e é inicialmente um meio TIV homogêneo com grau de anisotropia fraca de $8 \%$. O eixo de simetria do meio foi rotacionado de $\theta=30^{\circ}$ em relação ao eixo $x$. A matriz não rotacionada do tensor elástico normalizado pela densidade, $A_{i j}$, em $(\mathrm{km} / \mathrm{s})^{2}$ do meio é descrito da seguinte 
forma:

$$
A_{i j}=\left[\begin{array}{cccccc}
15.71 & 5.05 & 4.46 & & & \\
& 15.71 & 4.46 & & & \\
& & 13.39 & & & \\
& & & 4.98 & & \\
& & & & 4.98 & \\
& & & & & 5.33
\end{array}\right]
$$

A segunda camada é uma meio isotrópico homogêneo de $2 \mathrm{~km}$ de espessura, na qual é caracterizada pelas velocidades das ondas $\mathrm{P}$ e $\mathrm{S}$ normalizadas pela densidade, $\alpha=4,0 \mathrm{~km} / \mathrm{s}$ e $\beta=2,35 \mathrm{~km} / \mathrm{s}$, respectivamente.

Para simular um poço direcional, o poço foi considerado ter parte vertical e parte horizontal. A parte vertical do poço está localizada no centro do modelo e na direção do eixo $z$, estando em seu interior 2 receptores a $0,2 \mathrm{~km}$ e a $0,5 \mathrm{~km}$ de profundidade. Este segundo receptor também pertence a parte do poço horizontal que é orientado na direção do eixo $x$, estando espaçados em $0,3 \mathrm{~km}$ do terceiro receptor que está no interior do poço horizontal.

No levantamento foram utilizadas 180 fontes, distribuídas em cinco perfis cujo azimutes variam de $0^{\circ}$ a $360^{\circ}$ com incremento de $72^{\circ}$. O meio de cada perfil intercepta o centro do modelo. As fontes na aquisição do poço vertical são espaçadas regularmente por $0,1 \mathrm{~km}$ ao longo de cada perfil, começando pelo centro do modelo, enquanto que no poço horizontal, as fontes são espaçadas regularmente por $0,05 \mathrm{~km}$.

\section{Estimativa dos parâmetros WA}

A análise e estimativa dos parâmetros $W A$ adquiridos na inversão dos dados sintéticos medidos nos receptores 1 , 2 e 3 foram feitas através da comparação de projeções estereográficas da aproximação de primeira ordem da velocidade de fase (Pšenč I' k and Gajewski, 1998) determinada a partir dos parâmetros WA exatos e estimados (Fig. 2). A fórmula de primeira ordem da velocidade de fase é dada por:

$$
c\left(x_{m}, n_{j}\right)=\sqrt{\alpha^{2}+B_{33}},
$$

em que $B_{33}$ é a matriz dada na equação (1). A velocidade de fase $c\left(x_{m}, n_{j}\right)$ é função do vetor $\mathbf{n}=\left(n_{1}, n_{2}, n_{3}\right)$ e do vetor de parâmetros $\mathbf{x}$.

Através da análise da fórmula aproximada da velocidade de fase para ondas $P$ direta, foi possível determinar quantos e quais parâmetros $W A$ foram estimados nos receptores do poço direcional (Fig. 3, 4 e 5). Para se analisar a estabilidade da estimativa desses parâmetros foram feitas 500 simulações, onde os dados observados nos receptores 1, 2 e 3 estavam contaminados com ruído Gaussiano. Essas simulações foram utilizadas na inversão das equações (4) e (5) e, a partir delas, calculou-se a média e desvio padrão amostral das estimativas dos parâmetros $W A$.

$$
\begin{aligned}
\overline{x_{j}} & =\frac{\sum_{k=1}^{N_{s}}\left(\tilde{x_{j}}\right)^{k}}{N_{s}}, \\
\bar{v}_{j} & =\sqrt{\frac{\sum_{k=1}^{N_{s}}\left(\tilde{x_{j}}\right)^{k}}{N_{s}}-\left[N_{s} \bar{x}_{j}\right]^{2}},
\end{aligned}
$$

em que $N_{s}$ é o número de simulações numéricas $\left(N_{s}=500\right)$; $\tilde{x_{j}}$ são as estimativas dos parâmetros; o índice $j$ refere- se aos parâmetros $(j=1, \ldots, 15) ; \overline{x_{j}}$ e $\overline{v_{j}}$ são, respectivamente, o valor médio amostral e a variação amostral da estimativa dos parâmetros, $x_{j}$. A análise e precisão do mergulho, $\theta$, será feita da mesma forma que para os parâmetros $W A$ no decorrer do trabalho.

A Figura 2 mostra o estereograma da velocidade de fase calculada através da equação (11) com os parâmetros WA exatos e estimados, além da diferença percentual relativa entre eles. Nessa figura, percebe-se que as estimativas dos parâmetros WA apresentam bons resultados na determinação da velocidade de fase dos três receptores. $O$ erro nos três casos é inferior a 4\%. Já as Figuras 3,4 e 5 mostram quais parâmetros são bem estimados em cada receptor. 

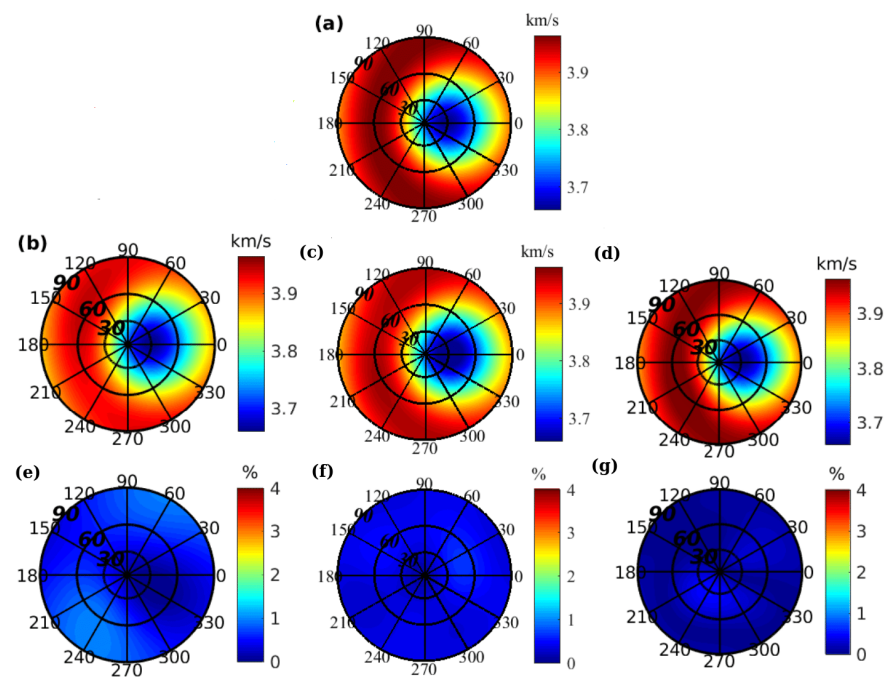

Figure 2: Projeções estereográficos para o receptor 1, 2 e 3. Sendo: (a) velocidade de fase computado usando parâmetros de WA exatos; (b), (c) e (d) velocidade da fase calculada usando parâmetro WA estimados para o receptor 1, 2 e 3, respectivamente; (e) erro percentual entre (a) e (b); (f) erro percentual entre (a) e (c); e (g) Erro percentual entre (a) e (d).

De acordo com a Figura 3, os parâmetros bem estimados na região vertical do poço (receptor 1) são: $A_{33}, A_{14}+2 A_{56}$, $A_{25}+2 A_{46}, A_{15}, A_{24}, A_{34}$ e $A_{35}$. Enquanto que na região horizontal (receptor 3 ), os parâmetros $W A$ com melhor precisão são: $A_{11}, A_{25}+2 A_{46}, A_{36}+2 A_{45}, A_{15}, A_{16}, A_{26} \mathrm{e}$ $A_{35}$ (Fig. 5).

Como o receptor 2 está localizado tanto na parte vertical quanto horizontal do poço, o resultado para este receptor foi obtido a partir junção dos parâmetros que são bem estimados usando tanto a equação do poço vertical (eq.4) quanto a equação do poço horizontal (eq.5), e os outros parâmetros são obtidos a partir da média (Fig. 4).

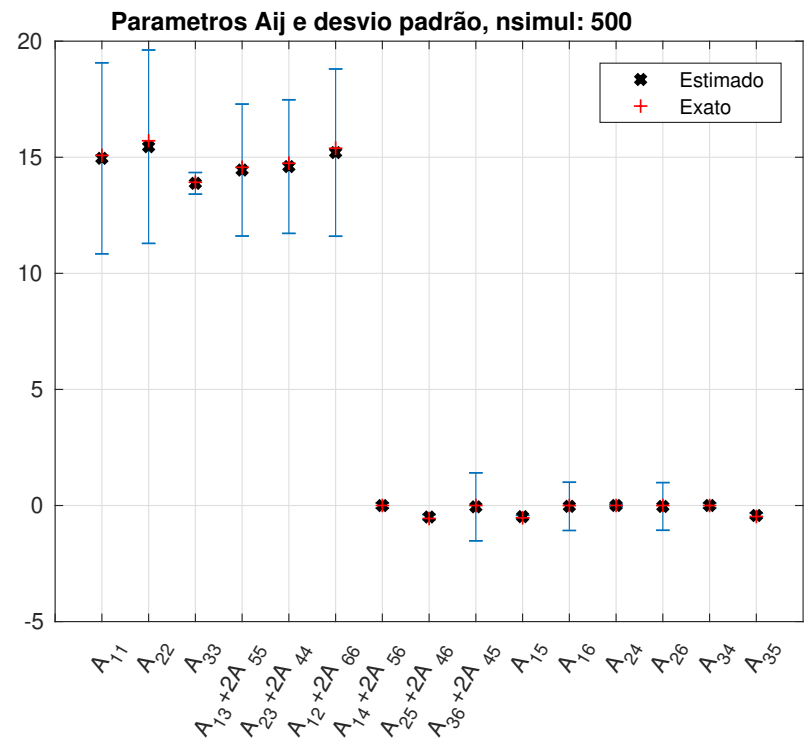

Figure 3: Gráfico que mostram os parâmetros WA $\left(A_{i} j\right)$ ) exatos, estimados e o desvio padrão deles do receptor 1

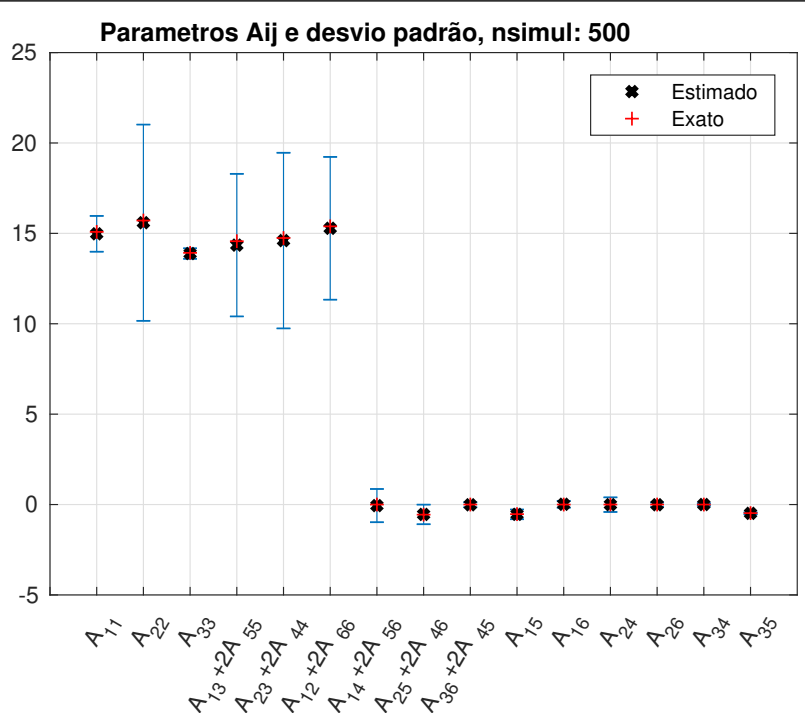

Figure 4: Gráfico que mostram os parâmetros WA $\left.\left(A_{i} j\right)\right)$ exatos, estimados e o desvio padrão deles do receptor 2

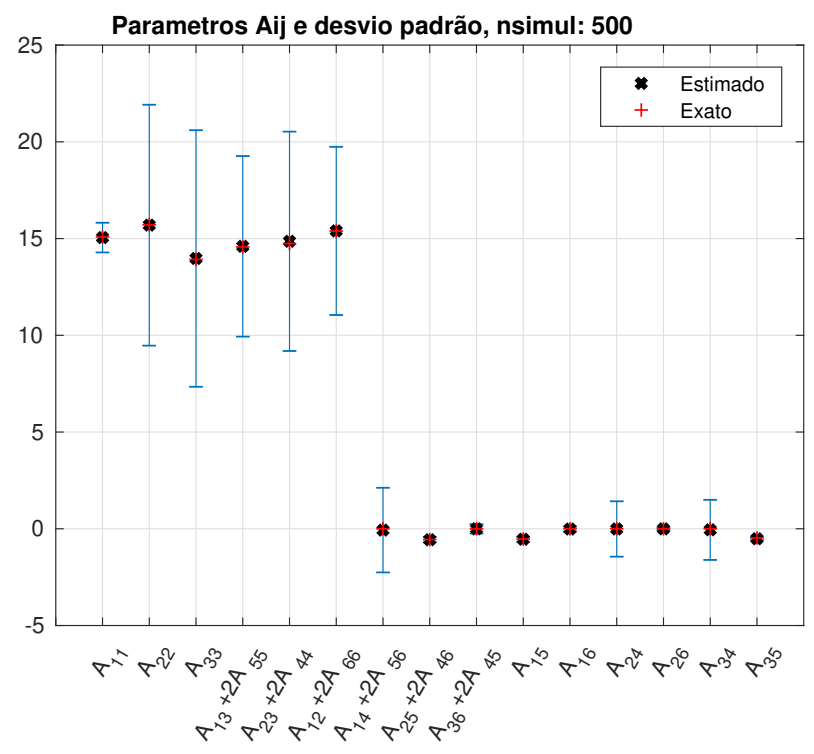

Figure 5: Gráfico que mostram os parâmetros $\left.W A\left(A_{i} j\right)\right)$ exatos, estimados e o desvio padrão deles do receptor 3

A próxima etapa do trabaho é a estimativa da orientação do eixo de simetria do meio TI. Essa estimativa depende de um grupo parâmetros bem estimada em poços constituídos de parte vertical e parte horizontal.

\section{Conclusão}

Neste trabalho foi feita a estimativa dos parâmetros de fraca anisotropia em um meio TI. É mostrado que dos 15 parâmetros que descrevem a propagação da ondas $q P$, apenas um grupo pequeno destes parâmetros é bem estimado para um poço vertical ou horizontal. Utilizando o tipo de modelagem apresentada neste trabalho pretendese estimar a orientação do eixo de simetria utilizando os parâmetros com as melhores estimativas no poço vertical em conjunto com os parâmetros com as melhores estimativas em um poço horizontal. 


\section{References}

Program ANRAY - Version 4.50:, 2019. Available on:http://sw3d.cz/software/sw3dcd8/anray/anray.htm. Access on: january 10th, 2019.

Barreto, A., Gomes, E., Macambira, R., and Costa, J., 2013, Improvement of local anisotropy estimation from vsp data through experimental design: Journal of Geophysics and Engineering, 10, no. 4, 045008.

Farra, V., and Pšenčık, I., 2003, Properties of the zeroth, first-, and higher-order approximations of attributes of elastic waves in weakly anisotropic media: The Journal of the Acoustical Society of America, 114, no. 3, 13661378.

Macambira, R. d. N. A., Gomes, E. d. N. S., and Barreto, A C. R., 2014, Analysis of a linear scheme for estimation of local anisotropy from p-wave data in multi-azimuth vsp surveys: Revista Brasileira de Geofísica, 32, no. 4, 707720.

Menke, W., 2018, Geophysical data analysis: Discrete inverse theory: Academic press.

Pšenč I' k, I., and Gajewski, D., 1998, Polarization, phase velocity, and nmo velocity of qp-waves in arbitrary weakly anisotropic media: Geophysics, 63, no. 5, 1754-1766.

Schoenberg, M., and Douma, J., 1988, Elastic wave propagation in media with parallel fractures and aligned cracks: Geophysical prospecting, 36, no. 6, 571-590.

Silva, B. d. S., 2018, Local anisotropy estimation from vsp data: analysis of $3 \mathrm{~d}$ survey design: Master's thesis, Universidade Federal do Pará, Brasil.

Thomsen, L., 1986, Weak elastic anisotropy: Geophysics, 51, no. 10, 1954-1966.

Zheng, X., and Pšenčík, I., 2002, Local determination of weak anisotropy parameters from qp-wave slowness and particle motion measurements: Seismic Waves in Laterally Inhomogeneous Media, 1881-1905. 